\title{
Functional Consequences of Neurite Orientation Dispersion and Density in Humans across the Adult Lifespan
}

\author{
(1)Arash Nazeri, ${ }^{1,2}$ M. Mallar Chakravarty, ${ }^{1,2,3,4}$ David J. Rotenberg, ${ }^{1}$ Tarek K. Rajji, ${ }^{2,5,6}$ (Y) Yogesh Rathi, ${ }^{7}$ \\ Oleg V. Michailovich, ${ }^{8}$ and Aristotle N. Voineskos ${ }^{1,2,5,6}$ \\ ${ }^{1}$ Kimel Family Translational Imaging-Genetics Laboratory, Research Imaging Centre, Campbell Family Mental Health Institute, Centre for Addiction and \\ Mental Health, Toronto, Ontario, Canada M5T 1R8, ${ }^{2}$ Department of Psychiatry, University of Toronto, Toronto, Ontario, Canada M5T 1R8, ${ }^{3}$ Cerebral \\ Imaging Centre, Douglas Institute, Verdun, Quebec, Canada H4H 1R3, ${ }^{4}$ Departments of Psychiatry and Biomedical Engineering, McGill University, \\ Montreal, Quebec, Canada H3A 2B4, Institute of Medical Science, University of Toronto, Toronto, Ontario, Canada M5S 1A8, ${ }^{6} \mathrm{Geriatric}$ Mental Health \\ Service, Centre for Addiction and Mental Health, Toronto, Ontario, Canada M6J 1H4, ${ }^{7}$ Laboratory of Mathematics in Imaging, Psychiatry Neuroimaging \\ Laboratory, Brigham and Women's Hospital, Harvard Medical School, Boston, Massachusetts 02215, and ${ }^{8}$ Department of Electrical and Computer \\ Engineering, University of Waterloo, Waterloo, Ontario, Canada N2L 3G1
}

As humans age, a characteristic pattern of widespread neocortical dendritic disruption coupled with compensatory effects in hippocampus and other subcortical structures is shown in postmortem investigations. It is now possible to address age-related effects on gray matter (GM) neuritic organization and density in humans using multishell diffusion-weighted MRI and the neurite-orientation dispersion and density imaging (NODDI) model. In 45 healthy individuals across the adult lifespan (21-84 years), we used a multishell diffusion imaging and the NODDI model to assess the intraneurite volume fraction and neurite orientation-dispersion index (ODI) in GM tissues. We also determined the functional correlates of variations in GM microstructure by obtaining resting-state fMRI and behavioral data. We found a significant age-related deficit in neocortical ODI (most prominently in frontoparietal regions), whereas increased ODI was observed in hippocampus and cerebellum with advancing age. Neocortical ODI outperformed cortical thickness and white matter fractional anisotropy for the prediction of chronological age in the same individuals. Higher GM ODI sampled from resting-state networks with known age-related susceptibility (default mode and visual association networks) was associated with increased functional connectivity of these networks, whereas the task-positive networks tended to show no association or even decreased connectivity. Frontal pole ODI mediated the negative relationship of age with executive function, whereas hippocampal ODI mediated the positive relationship of age with executive function. Our in vivo findings align very closely with the postmortem data and provide evidence for vulnerability and compensatory neural mechanisms of aging in GM microstructure that have functional and cognitive impact in vivo.

Key words: cognitive aging; diffusion-weighted MRI; GBSS; gray matter; neurite orientation dispersion; structure-function relationship

\section{Introduction}

Dendrites are key sites for synaptic integration and neuronal connectivity in the brain (Spruston, 2008). Postmortem studies have demonstrated that morphological alterations in dendritic structures are hallmarks of aging in gray matter (GM) (Dickstein et al., 2013) characterized by reduced complexity and regression of the dendritic tree in the neocortex (de Brabander et al., 1998; Duan et al., 2003). In contrast, dendritic growth and increased dendritic

Received Sept. 25, 2014; revised Dec. 6, 2014; accepted Dec. 10, 2014.

Author contributions: A.N., M.M.C., D.J.R., Y.R., O.V.M., and A.N.V. designed research; A.N. and A.N.V. performed research; A.N. analyzed data; A.N., M.M.C., D.J.R., T.K.R., Y.R., O.V.M., and A.N.V. wrote the paper.

A.N.V. is funded by the Canadian Institutes of Health Research, Ontario Mental Health Foundation, NARSAD, and the National Institute of Mental Health, National Institutes of Health (Grants R01MH099167 and R01MH102324). M.M.C. is funded by the W. Garfield Weston Foundation and Natural Sciences and Engineering Research Council. A.N. is a recipient of a fellowship from the Centre for Addiction and Mental Health.

The authors declare no competing financial interests.

Correspondence should be addressed to Dr. A.N. Voineskos, Kimel Family Translational Imaging-Genetics Laboratory, Research Imaging Centre, Campbell Family Mental Health Institute, Centre for Addiction and Mental Health, 250 College Street, Toronto, Ontario, Canada M5T1R8. E-mail: Aristotle.Voineskos@camh.ca.

DOI:10.1523/JNEUROSCI.3979-14.2015

Copyright $\odot 2015$ the authors $\quad 0270-6474 / 15 / 351753-10 \$ 15.00 / 0$ complexity occur within paleocortex (parahippocampal gyrus) and archicortex (hippocampus) in successful human aging (Buell and Coleman, 1979; Flood et al., 1985) and senescent animals (Pyapali and Turner, 1996).

GM structure has been extensively assessed in vivo at a macroscopic scale. However, in humans, the evaluation of GM microstructure, and dendritic organization in particular, is typically limited to postmortem tissue. These studies are generally confounded by progressive alterations that occur during the postmortem interval before tissue fixation (Penzes et al., 2011). In addition, postmortem studies typically obviate any possibility of meaningfully studying structure-function relationships.

Recent advances in diffusion-weighted MRI have opened new vistas to examine brain tissue microstructure in vivo (Assaf et al., 2013). These techniques use the diffusion properties of water molecules to estimate underlying cellular microstructural properties of brain tissue in the context of specific biophysical models. Neurite-orientation dispersion and density imaging (NODDI) is a recently proposed model that extends application of diffusion imaging from white matter (WM) to the GM tissue microstruc- 
Table 1. Demographic characteristics of participants $(n=45)$

\begin{tabular}{lc}
\hline Age (y), range & $53.9(18), 21-84$ \\
Sex (F/M) & $24 / 21$ \\
Handedness (R/L) & $43 / 2$ \\
Education (y) & $15.5(2.4)$ \\
WTAR IQ & $118.2(7.7)$ \\
MMSE & $29.3(1.0)$ \\
Diastolic BP (mmHg) & $74.7(9.6)$ \\
Systolic BP (mmHg) & $122.4(15.8)$ \\
BMI & $26.2(5.8)$ \\
CIRS-G (ratio score) & $1.61(0.58)$ \\
\hline
\end{tabular}

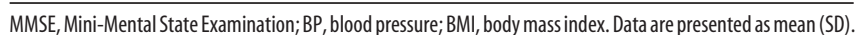

ture (Zhang et al., 2012). NODDI indexes neuritic density and dispersion of neurites in the brain tissue with intraneurite volume fraction $\left(\nu_{\mathrm{IN}}\right)$ and orientation dispersion index (ODI), respectively. These indices can be used to assess dendritic organization in vivo (Jespersen et al., 2007; Zhang et al., 2012). Others have attempted to investigate different aspects of GM microstructure using myelin mapping (Glasser and Van Essen, 2011; Grydeland et al., 2013) and diffusion tensor imaging (DTI) (Ball et al., 2013; Pereira et al., 2014) and, more recently, NODDI indices (Winston et al., 2014). However, the NODDI model (unlike DTI) fully accounts for effects of free-water contamination, which is of particular relevance in aging (Rathi et al., 2014).

As a primary aim, we sought to investigate regional patterns of GM microstructure in healthy humans across the adult life. We adopted a GM-based spatial statistics (GBSS) approach (Ball et al., 2013) for voxelwise analysis of NODDI-derived indices within the GM, including enhanced registration steps and customization to take full advantage of the NODDI model. We hypothesized that consistent with the postmortem data neocortical vulnerability and compensatory effects in hippocampal GM microstructure would be observed. In addition, recent evidence suggests that there is a close coupling between dendritic activity and blood-oxygen-level-dependent signal variation (Viswanathan and Freeman, 2007). Many studies find effects of age in brain networks using resting-state functional connectivity; however, whereas certain networks show age-related differences more often than others, there is no established consensus regarding the network or networks that are consistently affected (Ferreira and Busatto, 2013). Therefore, we also aimed to uncover the functional consequences of age-related GM microstructural variation using fMRI approaches as well as assessment of cognitive performance in the same individuals.

\section{Materials and Methods}

Participants. Forty-five healthy participants across the adult lifespan (21-84 years of age; female/male: $24 / 21$ ) were recruited at the Centre for Addiction and Mental Health (CAMH) in Toronto, Canada via referrals, study registries, and advertisements. All participants completed the Structured Clinical Interview for DSM-IV Disorders, the Mini-Mental Status Examination, and a urine toxicology screen. Exclusion criteria were any history of a mental disorder (including a dementia, current substance abuse, or a lifetime substance dependence, except for simple phobias); positive urine toxicology, a first-degree relative with a history of psychotic mental disorder, a history of head trauma with loss of consciousness, seizure, or another neurological disorder. Participants were characterized with the Wechsler Test for Adult Reading (WTAR); Edinburgh handedness inventory (Oldfield, 1971); Hollingshead index; Clinical Illness Rating Scale for Geriatrics (CIRS-G) (Miller et al., 1992); weight, height, and blood pressure (Table 1). The study was approved by the Research Ethics Board of CAMH and all participants provided written informed consent.

All participants underwent a battery of cognitive testing. Raw cognitive scores were converted to $z$-scores. Performance in working memory/processing speed was computed as the average of standardized scores from: Stroop ratio index (ratio of color-word time score to color time score; Trenerry et al., 1989), Trail-Making Test B (Reitan and Wolfson, 1985), and the Letter-Number Sequencing Test (Wechsler, 1997).

Imaging protocol. Diffusion, structural, and functional images were acquired for all participants on a 3 T Discovery MR750 system (General Electric) equipped with an 8-channel head coil. High-resolution $\mathrm{T}_{1}$-weighted anatomical images were acquired using a $3 \mathrm{D}$ inversionprepared fast spoiled gradient-recalled echo acquisition, FSPGRBRAVO $(\mathrm{TE} / \mathrm{TR}=3 / 6.7 \mathrm{~ms})$ with an isotropic voxel size of $0.9 \times 0.9 \times$ $0.9 \mathrm{~mm}^{3}$. For the diffusion imaging, a multishell protocol was acquired along 30 noncollinear directions at $3 b$-values $\left(1000,3000,4500 \mathrm{~s} / \mathrm{mm}^{2}\right)$ in addition to 15 interspersed $b=0$ images using a single-shot echoplanar sequence. To increase the signal-to-noise ratio, the high $b$-value images $\left(b=4500 \mathrm{~s} / \mathrm{mm}^{2}\right)$ were acquired with NEX $=2$. The acquisition parameters were as follows: $\mathrm{TE} / \mathrm{TR}=108 / 12,000 \mathrm{~ms}$, voxel dimension of $2 \times 2 \times 2 \mathrm{~mm}^{3}, 82$ slices. Resting-state functional scans were acquired using an axial spiral fMRI acquisition with the following parameters: $\mathrm{TE} / \mathrm{TR}=30 / 2000 \mathrm{~ms}, 210$ volumes, voxel dimension of $3.4 \times 3.4 \times 5$ $\mathrm{mm}^{3}, 31$ slices. During the resting-state fMRI, participants were requested to close their eyes and let their mind wander.

Diffusion MRI processing. Eddy current-induced distortions and motion-related misalignment of diffusion images were corrected using FMRIB's Software Library (FSL) version 5.0.6 (Jenkinson et al., 2012). After brain extraction (Smith, 2002), the diffusion tensor model was fitted in each voxel using the dtifit function to estimate fractional anisotropy (FA) maps.

In the NODDI model, three microstructural environments are distinguished (intracellular, extracellular, and CSF compartments; Zhang et al., 2012). The intracellular component models dendrites and axons as a set of sticks with restricted diffusion perpendicular to neurite axes and unhindered diffusion along them (Sotiropoulos et al., 2012). NODDI adopts the Watson distribution to model distribution of these sticks (neurites), which permits modeling of highly dispersed neuritic structures such as dendritic trees in the GM tissue (Zhang et al., 2012). NODDI generates five independent parametric maps, three of which were used in this study: (1) ODI, which is the dispersion coefficient of the sticks (neurites) and ranges from 0 for no dispersion to 1 for fully dispersed; (2) $\nu_{\mathrm{IN}}$, which indexes the fraction of tissue volume restricted within neurites, $(3)$ fraction of $\operatorname{CSF}\left(f_{\mathrm{CSF}}\right)$, which indexes percentage of the volume in each voxel that is occupied by free water. The NODDI model was fitted using the NODDI toolbox (https://www.nitrc. org/projects/noddi_toolbox/) in the MATLAB environment.

GM-based spatial statistics. We adopted GM-based spatial statistics (GBSS) methodology (Ball et al., 2013) to investigate effects of age on GM in a voxelwise fashion. As discussed below, the registration steps were enhanced and customized to exploit the potential of the NODDI modeling. The processing pipeline is summarized in Figure 1. As in other voxel-based approaches, accurate cross-subject alignment of anatomically related regions is of paramount importance. This issue is even more critical with diffusion imaging analysis of the cortical regions (cerebellar and cerebral cortex), given the relatively thin cortical structures and sensitivity of diffusion metrics to partial volume contamination (Koo et al., 2009). To overcome these caveats, GBSS adapts the tract-based spatial statistics (TBSS) (Smith et al., 2006) framework for GM analysis by skeletonizing the GM and projecting diffusion metrics (e.g., FA) from the most probable local GM voxel on to the skeleton for group comparison.

Unlike the original GBSS pipeline, in which tissue classification takes place in the structural images (Ball et al., 2013), we directly segmented brain tissues using the diffusion data as follows: (1) CSF partial volume in each voxel was directly estimated using the NODDI model parameter, $f_{\text {CSF }}$ (Zhang et al., 2012); (2) WM segmentation was performed on the FA images using Atropos (Avants et al., 2011a) for two class classification (WM/non-WM) to derive fraction of WM $\left(f_{\mathrm{WM}}\right)$ (it has been previously shown that FA images can be used to robustly classify WM tissue; Liu et al., 2007; Kumazawa et al., 2010); and (3) fraction of GM ( $\left.f_{\mathrm{GM}}\right)$ in each 

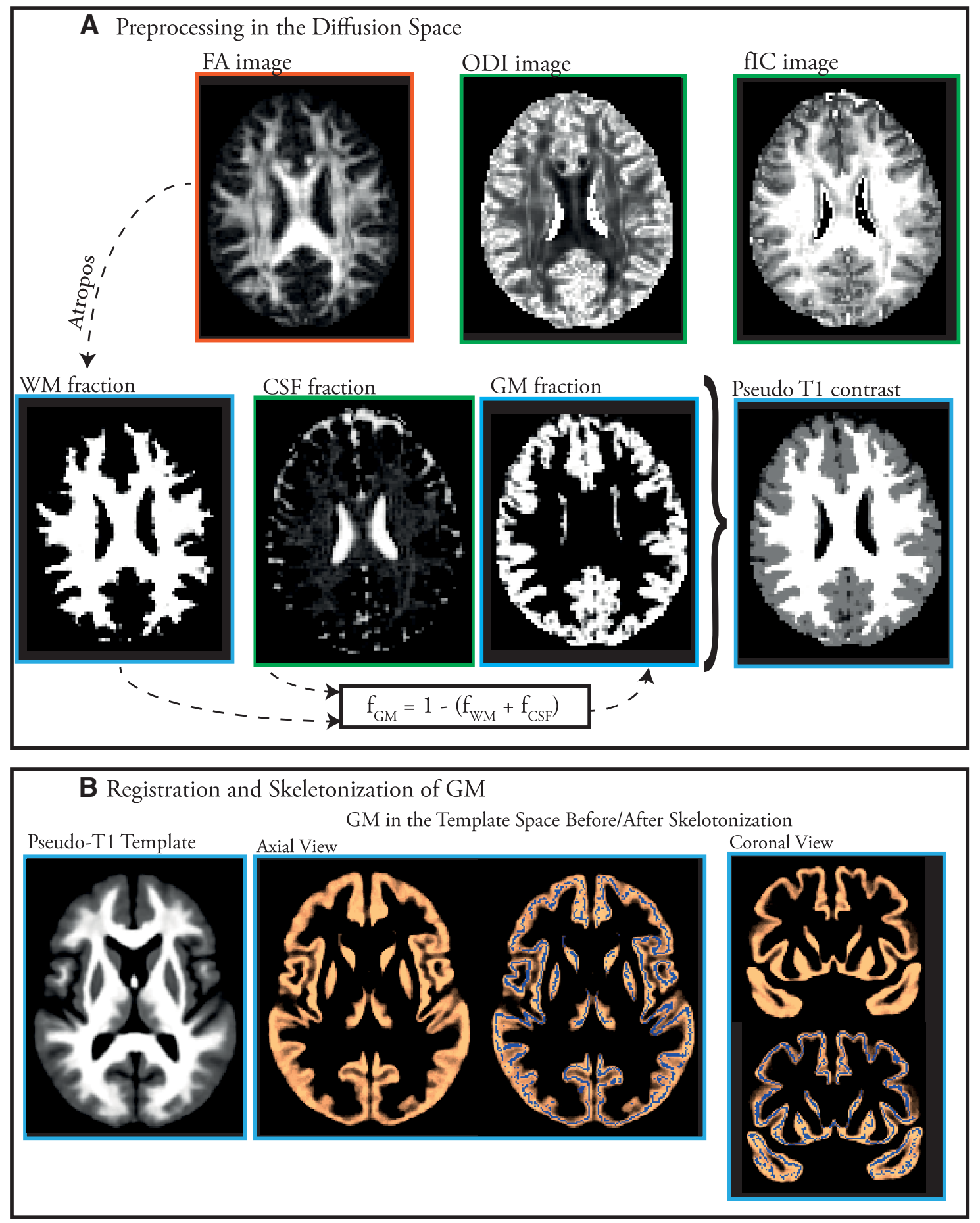

Figure 1. Overview of the pipeline proposed in this study. $A$, Diffusion image processing steps. FA maps were created by fitting the DTI model (orange box); the CSF fraction, ODI, and neurite density $\left(\nu_{|N|}\right)$ images were generated by applying the NODDI model to the diffusion images (green boxes). WM and GM fraction were estimated indirectly. Finally, images with a pseudo-T1 contrast were generated for registration steps. $\boldsymbol{B}$, Registration and skeletonization of GM. A norm-template was created by iterative nonlinear registration of pseudo-T1 images.

voxel was determined by simply subtracting fractions of CSF and WM from 1 as follows:

$$
f_{\mathrm{GM}}=1-\left(f_{\mathrm{CSF}}+f_{\mathrm{WM}}\right)
$$

Diffusion parameter maps do not have sufficient gray/white/CSF contrast to allow for accurate registration of cortical GM. Therefore, we generated maps with a contrast similar to T1 images (pseudo-T1 images) by multiplying partial volume estimations of each tissue class by their corresponding contrast (contrasts for $\mathrm{CSF}=0, \mathrm{GM}=1, \mathrm{WM}=2$ ). For groupwise nonlinear registration, we used the buildtemplateparallel.sh script in the ANTS software package version 1.9 (Avants et al., 2011b), which has recently been shown to significantly improve registration of diffusion images (Schwarz et al., 2014). Aligned GM probability maps were averaged across individuals and thinned (skeletonized) so that the skeleton represents the center of highly probable GM voxels. Each subject's (aligned) ODI, $\nu_{\mathrm{IN}}$, and $f_{\mathrm{GM}}$ image were then projected onto the skeleton. This is achieved, for each skeleton voxel, by searching perpendicular to the skeleton structure for the most probable local GM voxel (Ball et al., 2013). Only voxels with $f_{\mathrm{GM}}>0.7$ in $>75 \%$ of the subjects were retained in the skeleton. The remaining voxels with nonsatisfactory 
$f_{\mathrm{GM}}<0.7$ were excluded from the analysis using the setup_masks script, implemented in FSL.

Region of interest analysis. Lobar (frontal, occipital, parietal, temporal), striatal, and cerebellar regions of interest (ROIs) from the MNI atlas, along with hippocampal mask from the Harvard Oxford subcortical atlas (http://fsl.fmrib.ox.ac.uk/fsl/fslwiki/Atlases) were transformed to individual diffusion spaces by applying inverse warp fields generated during ANTS nonlinear registration step. The $f_{\mathrm{GM}}$ images in the diffusion native space were thresholded at 0.7 and binarized. The resulting GM masks were multiplied by the transformed ROIs. Finally, average ODIs were extracted separately from each ROI.

To control for the effects of cortical thickness and subcortical volume, cortical surface reconstruction, cortical thickness measurement, and subcortical segmentation were performed on structural $\mathrm{T}_{1}$ images using the FreeSurfer toolkit version 5.1 (Dale et al., 1999; Fischl and Dale, 2000; Fischl, 2012). Average lobar cortical thickness and subcortical volume were recorded for each individual.

For WM analysis, all FA images were nonlinearly transformed to the MNI space and then averaged to create the mean FA map. Next, a skeletonization procedure was applied to the resulting mean FA image (Smith et al., 2006). Non-WM voxels were discarded from the skeleton by thresholding the skeletonized mean FA (FA >0.2) (Smith et al., 2006). To account for residual misalignments and to facilitate groupwise comparison, each individual's FA image was searched orthogonal to the skeleton to find the local maxima. These local maxima were then projected back onto the skeleton. Finally, average FA was extracted from each skeletonized lobar ROI.

Resting-state fMRI analysis. Functional image preprocessing and analysis were conducted using FSL version 5.0.6 (Jenkinson et al., 2012). Functional data preprocessing included: discarding the first 5 volumes, motion correction with MCFLIRT, brain extraction using BET (Smith, 2002), spatial smoothing with a Gaussian kernel $(\sigma=4 \mathrm{~mm})$, and highpass temporal filter with a $100 \mathrm{~s}$ cutoff. fMRI volumes were linearly registered to the individual's structural scan and nonlinearly transformed to the MNI space images using FNIRT. Next, functional images were denoised using FMRIB's independent component analysis (ICA)based Xnoiseifier (FIX) version 1.0.6 (Griffanti et al., 2014; SalimiKhorshidi et al., 2014). This algorithm provides an automatic solution for denoising fMRI data via accurate classification of components derived from ICA (Salimi-Khorshidi et al., 2014). Each individual's preprocessed functional volumes were submitted to single-session ICA using FSL MELODIC version 3.14 (Beckmann and Smith, 2004; Beckmann et al., 2005). The FIX algorithm was trained using a library of noise components (manually classified in 10 randomly selected subjects). Finally, the entire functional dataset was denoised by automatically classifying artifactual components.

For voxelwise comparisons of resting-state functional connectivity among subjects, we used the "dual regression" approach implemented in FSL (Filippini et al., 2009). Denoised functional data containing 205 time points for each subject were temporally concatenated across subjects. The resulting $4 \mathrm{D}$ dataset was submitted to groupwise ICA (http://fsl. fmrib.ox.ac.uk/fsl/fslwiki/MELODIC) with a dimensionality of 25 . The components that corresponded to previously described functional networks that involve neocortical regions were selected for further analysis (Smith et al., 2009). Six resting-state networks were identified. These included the primary visual network (corresponding to components $1_{20}$ and $2_{20}$ of 20-dimensional ICA resting-fMRI components available on http://www.fmrib.ox.ac.uk/analysis/brainmap + rsns), visual association network (corresponding to component $3_{20}$ ), default mode (DMN, corresponding to component $4_{20}$ ), sensorimotor network (corresponding to component $6_{20}$ ), right frontotemporal network (corresponding to component $9_{20}$ ), and left frontotemporal network (corresponding to component $10_{20}$ ) (Smith et al., 2009). Next, temporal dynamics for each independent component for each individual subject was determined using spatial regression. Finally, using temporal regression, the resulting time courses were regressed into the same $4 \mathrm{D}$ dataset to get a subjectspecific set of spatial connectivity maps.

For each of these networks, average neocortical ODI was extracted using a similar approach as described above for ROI analyses. Resting- state networks were thresholded at $z>3$, binarized, nonlinearly transformed to the diffusion space, and multiplied by the cortical mask derived from the Harvard-Oxford atlas.

Statistical analyses. Cross-subject voxelwise analyses were performed nonparametrically using Randomise (part of FSL, 5000 permutation; Nichols and Holmes, 2002) to test the effects of age independent of sex on GM microstructure (GBSS), the effects of ODI extracted from restingstate networks on functional connectivity of the corresponding network while controlling for effects of age and sex (dual regression), and the effects of age on functional connectivity of each resting-state network while controlling for sex (dual regression). Threshold-free cluster enhancement was used to provide brain-wide significance without defining an arbitrary cluster-forming threshold (Smith and Nichols, 2009). A familywise error (FWE)-corrected $p\left(p_{\mathrm{FWE}}\right)<0.05$ was considered significant for GBSS. For dual regression analyses, statistical thresholds were determined by application of Bonferroni correction for 6 resting-state networks, where $p_{\text {FWE }}<0.0083$ (0.05/6) was considered significant.

To investigate the effect of age on regional ODI, ROI analyses were performed using general linear models in the $\mathrm{R}$ version 3.0.2 environment. To further evaluate the nonlinear effects of aging on ODI values extracted from cortical (frontal, occipital, parietal, and temporal lobe GM) and subcortical (cerebellum, hippocampus, and striatum) ROIs, regression with a restricted cubic spline function was conducted (rms package implemented in R). Statistical thresholds were determined by application of Bonferroni correction for 7 ROIs at which $p<0.0071$ $(0.05 / 7)$ was considered significant. To determine how well neocortical microstructure (ODI) predicts chronological age compared with the macrostructural properties of these structures (average cortical thickness) and lobar mean FA, root mean squared error (RMSE) and $R^{2}$ of the respective models were compared.

Effects of regional ODI (extracted from 48 cortical regions using Harvard-Oxford cortical atlas) on cognitive function were interrogated using stepwise forward-regression analysis, whereas accounting for effects of age and sex. Assuming that regional ODI would serve as mediators between age and cognitive performance, we conducted bootstrapping tests for mediation analysis, using the Preacher and Hayes (2008) SPSS macro with 5000 samples in the SPSS version 21.0 environment (Preacher and Hayes, 2008). This approach yields $\beta$ regression values for the indirect effect estimates and the corresponding $95 \%$ accelerated and bias-corrected confidence intervals (mediation effect is considered significant if the interval does not include 0 ).

\section{Results}

\section{Aging pattern of GM microstructure}

In a sample of 45 healthy subjects across the adult lifespan (21-84 years of age; female/male: $24 / 21$; Table 1 ), we found a widespread decrease in the neocortical-ODI $\left(p_{\mathrm{FWE}}<0.05\right)$ with advancing age. In contrast, only voxels in the cerebellar crus I/II demonstrated a significant age-related increase in ODI (Fig. 2, Table 2). No significant age-related difference was observed for GM $\nu_{\mathrm{IN}}$. When we repeated the analysis using different $f_{\mathrm{GM}}$ thresholds $\left(f_{\mathrm{GM}}>0.65\right.$ and $\left.f_{\mathrm{GM}}>0.75\right)$, similar patterns of lower neocortical ODI and higher cerebellar ODI were observed with older age (data not shown).

ROI analysis (Fig. 3) revealed significant linear decline with advancing age for frontal ODI $\left(t=-6.41, p=1.04 \times 10^{-7}\right)$, occipital ODI $\left(t=-5.66, p=1.23 \times 10^{-6}\right)$, parietal ODI $(t=$ $\left.-8.31, p=2.09 \times 10^{-10}\right)$, and temporal ODI $(t=-4.39 p=$ $\left.7.51 \times 10^{-5}\right)$, whereas a similar trend, albeit nonsignificant after multiple-comparison correction, was also found for striatum $(t=-2.56, p=0.014)$. In contrast, a significant linear agerelated increase in cerebellar $(t=3.25, p=0.0022)$ and hippocampal-ODI $(t=2.25, p=0.0071)$ was observed. In addition, hippocampal ODI demonstrated a significant nonlinear relationship with age (nonlinear term: $p=0.006$ ). These effects remained stable after inclusion of cortical thickness/subcortical 


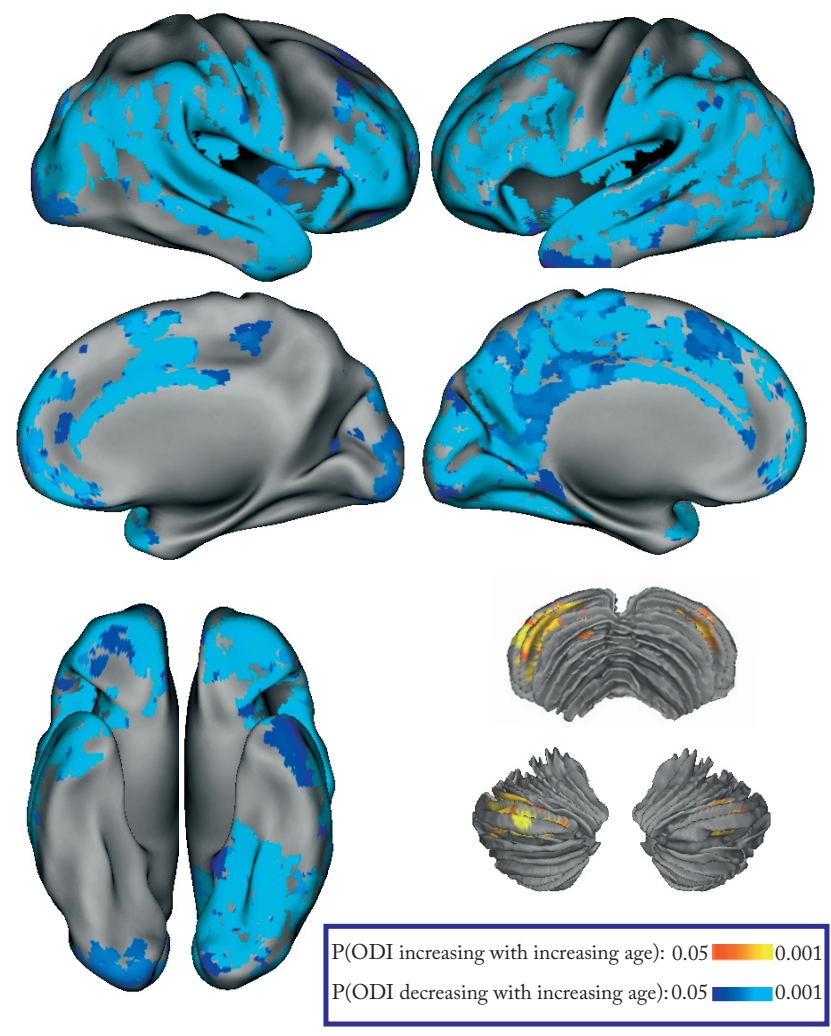

Figure 2. Effects of aging on regional GM microstructure. GBSS results demonstrating significant $\left(p_{\mathrm{FWE}}<0.05\right)$ ODI reduction with advancing age throughout neocortex and increased ODI in cerebellar lobule-VIIA.

volume measurements in the model (frontal lobe: $p=1.86 \times$ $10^{-5}$, occipital lobe: $p=1.2 \times 10^{-5}$, parietal lobe: $p=2.20 \times$ $10^{-8}$, temporal lobe: $p=0.0018$, hippocampus nonlinear effect: 0.008 , and cerebellum: $p=0.034)$. A more detailed analysis using 48 cortical ROIs revealed that ODI extracted from a majority of cortical regions (27 of 48) showed a significant decline with advancing age $(p<0.001$, after correction for multiple comparisons). A similar trend was observed in 10 additional cortical regions $(p<0.05)$.

Hippocampal ODI and cerebellar ODI showed negative correlation $(p<0.05)$ with hippocampal and cerebellar volumes, respectively, whereas frontal ODI and parietal ODI demonstrated a positive correlation with frontal and parietal cortical thickness measurements, respectively $(p<0.05)$. However, these apparently significant correlations disappeared after controlling for the effects of age.

\section{Prediction of chronological age}

The best prediction performance of chronological age was for the parietal ODI $\left(R^{2}=0.63, \mathrm{RMSE}=10.8 \mathrm{y}\right)$ followed by frontal ODI $\left(R^{2}=0.51\right.$, RMSE $\left.=12.5 y\right)$. For each lobar ROI, mean GM ODI consistently outperformed other measures of brain structure, namely the mean cortical thickness measurements and lobar WM fractional anisotropy for the prediction of chronological age (Table 3 ).

\section{GM microstructure in relation to brain resting-state functional connectivity}

In the same participants, we evaluated the effects of average GM-ODI extracted from neocortical regions of resting-state networks on the brain-wide functional connectivity of these net- works, whereas controlling for age and sex. From the group ICA, 6 of the 25 components (DMN, visual association, primary visual, sensorimotor, and left/right frontotemporal networks) were visually identified as corresponding to previously described functional networks that predominantly involve neocortical regions (Smith et al., 2009). A significant positive relationship between mean GM ODI extracted from the DMN and functional connectivity (temporal-coherence) between DMN and occipital cortex was observed ( $p=0.002$, Fig. 4 , Table 4 ). Similarly, higher GMODI extracted from the visual association network was associated with increased connectivity between this network and the medial aspect of the frontal lobe, corresponding to the anterior DMN ( $p=0.003$; Fig. 4, Table 4). GM-ODI extracted from sensorimotor and primary visual networks correlated positively, albeit nonsignificantly after multiple-comparison correction (sensorimotor network: $p=0.020$; primary visual network: $p=0.021$ ), with functional connectivity between these networks and insula, anterior and posterior cingulate cortices, and precuneus. In contrast, we did not detect any significant positive relationship between mean ODI extracted from the two lateralized frontotemporal networks (right and left) and functional connectivity of these networks. Furthermore, we found a tendency toward reduced functional connectivity between the right frontotemporal network and right frontal pole with higher ODI from this resting state network ( $p=0.027$; Table 4$)$.

We further investigated age-related differences in restingstate functional connectivity for each network (Fig. 4, Table 4). All four networks that showed higher functional connectivity with higher GM ODI also demonstrated age-related deficits in functional connectivity $\left(p_{\mathrm{FWE}}<0.05\right)$. No age effect was observed for the left and right lateralized frontotemporal networks. These age-related effects disappeared when effects of age were modeled in conjunction with GM ODI.

\section{Cognitive correlates of GM microstructure}

Across 48 cortical ROIs, frontal pole ODI was a significant determinant of working memory/processing speed independent of age (partial $r=0.49, p=0.0009$ ) after correction for multiple comparisons. In the follow-up analysis, hippocampal ODI $(t=2.05$, $p=0.047)$ also contributed positively to working memory/processing speed performance along with frontal pole ODI $(t=3.9$, $p=0.0004$ ) independent of age/sex. Consistent with our hypothesis, the mediation analysis demonstrated that frontal pole ODI mediated the negative relationship of age with cognition, whereas hippocampal-ODI mediated the protective effects of age (Fig. 5).

\section{Discussion}

By applying the NODDI model to multishell diffusion images, we found an in vivo pattern aligned very closely with published postmortem data indexing neocortical vulnerability and hippocampal compensation. We further demonstrated that these microstructural differences have consequences in cognitive function and brain resting-state networks with age-related susceptibility.

Effects of age on GM ODI were independent of GM volume/ cortical thickness measurements. Moreover, there were no associations between GM ODI and cortical thickness or subcortical volume after controlling for age. This suggests that GM microstructure (ODI) and macrostructure (volume/cortical thickness) likely represent independent processes in normal aging. Age trends for ODI exhibited substantial regional specificity and matched age-related dendritic changes previously documented in each region. Congruent with neocortical dendritic deficits with 
Table 2. MNI coordinates, volume, and cortical regions of GM clusters from GBSS analysis demonstrating significant ( $\left.p_{\text {FWE }}<0.05\right)$ effect of age on GM ODI

\begin{tabular}{|c|c|c|c|c|c|c|c|}
\hline \multirow[b]{2}{*}{ Cluster } & \multirow{2}{*}{$\begin{array}{l}\text { Volume } \\
\left(\mathrm{mm}^{3}\right)\end{array}$} & \multirow{2}{*}{$\begin{array}{l}\text { Minimum } \\
p \text {-value }\end{array}$} & \multicolumn{3}{|c|}{ MNI coordinates } & \multirow[b]{2}{*}{ Hemisphere } & \multirow[b]{2}{*}{ Brain regions } \\
\hline & & & $x$ & Y & Z & & \\
\hline \multicolumn{8}{|c|}{ ODI increase with age } \\
\hline $\mathrm{A} 1$ & 1087 & 0.005 & 36 & -71 & -38 & Right & Crus I, Crus II \\
\hline A2 & 303 & 0.015 & -29 & -74 & -38 & Left & Crus I, Crus II \\
\hline \multicolumn{8}{|c|}{ ODI decline with age } \\
\hline B1 & 6111 & 0.003 & 43 & 50 & -12 & Right & FPo, ACC, MFG, SFG \\
\hline B2 & 4984 & 0.004 & -1 & 4 & 35 & Left & Precuneus, PCC, ACC, PoCG, PreCG \\
\hline B3 & 7599 & 0.003 & 56 & -62 & -5 & Right & LOC, SMG, MTG, STG, PoCG, COC \\
\hline B4 & 2768 & 0.003 & 42 & 5 & -43 & Right & TPo, MTG \\
\hline B5 & 10669 & 0.003 & -39 & 48 & -13 & Left & SFG, MFG, IFG, OFC, FPo \\
\hline B6 & 7828 & 0.003 & -41 & -73 & -17 & Left & LOC, SMG, AG, Fus \\
\hline B7 & 2174 & 0.003 & -41 & 3 & -23 & Left & TPo, MTG \\
\hline
\end{tabular}

ACC, anterior cingulate cortex; $\mathrm{AG}$, angular gyrus; $\mathrm{COC}$, central opercular cortex; $\mathrm{OFC}$, orbitofrontal cortex; $\mathrm{FP}$, frontal pole; Fus, fusiform gyrus; IFG, inferior frontal gyrus; $\mathrm{LOC}$, lateral occipital cortex; MedFC, medial frontal cortex; $\mathrm{MTG}$, middle temporal gyrus; PoCG, postcentral gyrus; PreCG, precentral gyrus; PCC, posterior cingulate cortex; SMG, supramarginal gyrus; SFG, superior frontal gyrus; STG, superior temporal gyrus.

A Cortical Regions

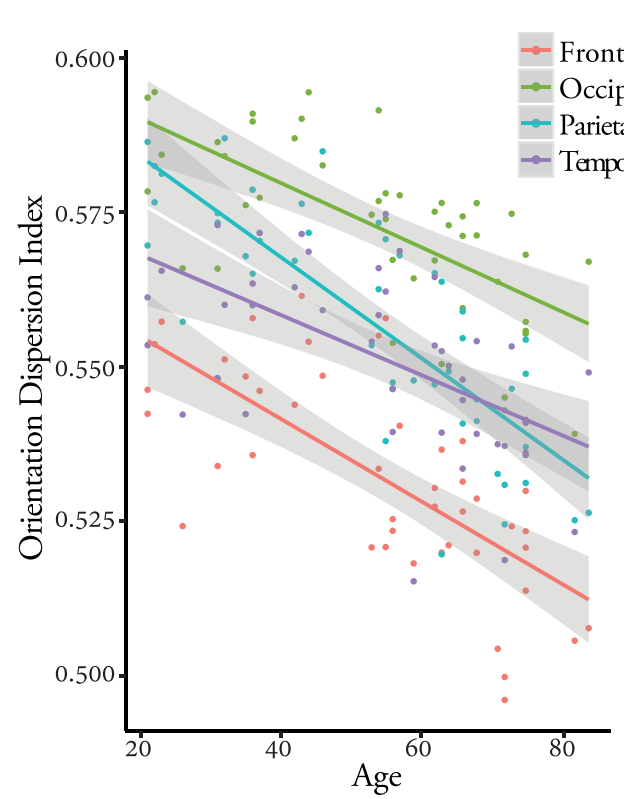

B Subcortical Regions

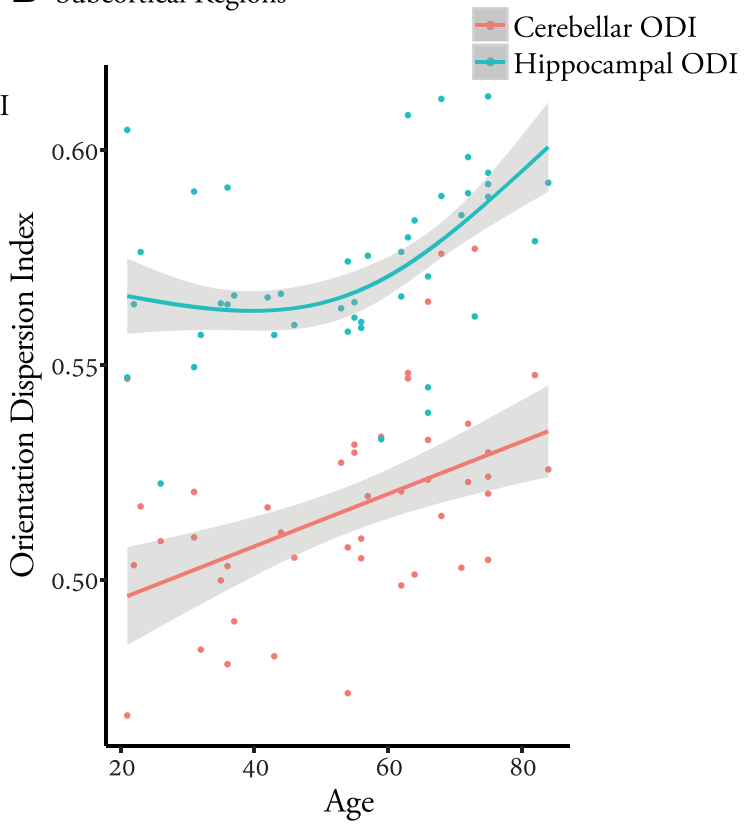

Figure 3. Cortical $(\boldsymbol{A})$ and subcortical (B) ROl analysis demonstrating significant age effects on regional ODI. A nonlinear fit for the hippocampal ODI-age relationship is shown given its significant nonlinear relationship with age.

Table 3. RMSE and $R^{2}$ of mean GM ODI, mean cortical thickness, and mean WM FA predicting chronological age

\begin{tabular}{llll}
\hline Lobar region & $\begin{array}{l}\text { Mean GM ODI } \\
\left(R^{2}, \text { RMSE }\right)\end{array}$ & $\begin{array}{l}\text { Mean cortical thickness } \\
\left(R^{2}, \text { RMSE }\right)\end{array}$ & $\begin{array}{l}\text { Mean WM FA } \\
\left(R^{2}, \text { RMSE }\right)\end{array}$ \\
\hline Frontal lobe & $0.51,12.5 \mathrm{y}$ & $0.34,14.4 \mathrm{y}$ & $0.18,16.1 \mathrm{y}$ \\
Occipital lobe & $0.45,13.2 \mathrm{y}$ & $0.16,16.2 \mathrm{y}$ & $0.13,16.6 \mathrm{y}$ \\
Parietal lobe & $0.63,10.8 \mathrm{y}$ & $0.19,16.0 \mathrm{y}$ & $0.12,16.6 \mathrm{y}$ \\
Temporal lobe & $0.33,14.5 \mathrm{y}$ & $0.17,16.2 \mathrm{y}$ & $0.15,16.4 \mathrm{y}$ \\
\hline
\end{tabular}

advancing age, decreased complexity, and regression of the dendritic tree (Duan et al., 2003; Dickstein et al., 2013), we observed pervasive age-related decline in neocortical ODI. Age-related deficits in frontoparietal ODI were more pronounced than those in temporo-occipital ODI in coherence with a retrogenesis pattern (i.e., GM regions that mature last are the first to be affected in later life; Reisberg et al., 1999). Only a small number of quantitative dendritic postmortem investigations (either in humans or in nonhuman primates) have studied regional specificity of aging in the neocortex (Jacobs et al., 1997; Young et al., 2014) because most studies have typically focused on only a single cortical area (Nakamura et al., 1985; Jacobs and Scheibel, 1993; Anderson and Rutledge, 1996; Duan et al., 2003). Future postmortem studies that examine the effect of aging simultaneously on multiple cortical regions throughout the neocortex would help to facilitate comparison between our in vivo results and postmortem findings (Kim et al., 2013).

We observed a possible protective effect of higher hippocampal ODI with advancing age. Intriguingly, age-related extension and growth of the dendritic tree have been reported in the hippocampus (Flood et al., 1985; Pyapali and Turner, 1996). These changes have been regarded as a compensatory mechanism that occurs in successful aging in response to partial deafferentation (Buell and Coleman, 1979; Pyapali and Turner, 1996). Our in vivo results agree with previous postmortem results and could be interpreted as age-related increases of hippocampal ODI playing a protective role against cognitive aging. In cerebellum, we found a nonsignificant $\left(p_{\mathrm{FWE}}=0.11\right.$ ) age-related decline of ODI within the vermal region. However, ODI increased significantly with age in the hemispheric regions of lobule VIIA (cerebro-cerebellum) 

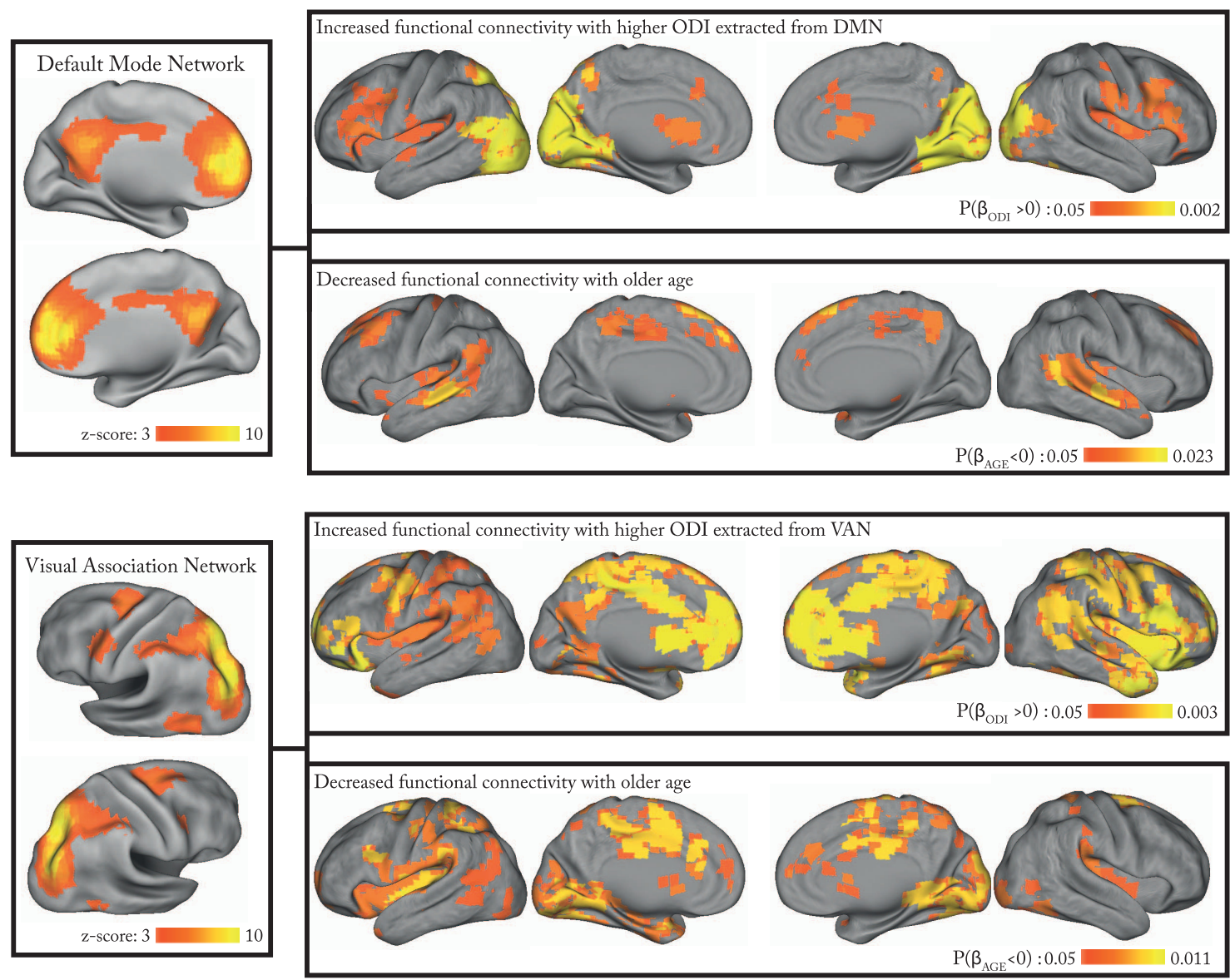

Figure 4. Effects of GM ODI extracted from DMN and visual association networks (VANs) on voxelwise functional connectivity (temporal coherence) of the corresponding network while controlling for effects of age and sex(dual regression). Age-related differences in resting-state functional connectivity for each network are also depicted for both networks.

(Schmahmann and Pandya, 1997). Virtually all previous studies examining the impact of aging on cerebellar dendritic organization have focused solely on the vermal region of the cerebellum and have observed age-related dendritic and synaptic deficits in this region (Rogers et al., 1984; Chen and Hillman, 1999), which is consistent with our results. Lobule VIIA, however, is an evolutionary recent area of the cerebellum that is densely connected to the neocortex (Schmahmann and Pandya, 1997). This suggests that ODI increases in the cerebellar hemispheres may also be a compensatory response to age-related deafferentation.

Higher GM ODI sampled from DMN and visual association network was associated with increased functional connectivity of these networks. A similar trend was also observed for primary visual and sensorimotor networks. We also observed age-related deficits in functional connectivity of networks that were positively correlated with GM ODI. There was partial anatomical overlap between effects of ODI and age on functional connectivity for each of these networks. Furthermore, age-related effects disappeared when age was modeled together with GM ODI. These findings collectively suggest that GM ODI partially mediates the effects of age on resting-state functional connectivity.

Functional connectivity disruption of the DMN with advancing age is a well replicated fMRI finding in healthy aging (Mowinckel et al., 2012; Ferreira and Busatto, 2013). Our findings demonstrate how both intrinsic (within the DMN itself) and extrinsic (within other resting-state networks) microstructural GM differences could contribute to functional connectivity variations of the DMN. In addition, we observed that GM ODI was related to functional connectivity of resting-state networks with several regions outside of the boundaries of these networks. Permutation testing after dual regression analysis permits identification of any brain region where functional connectivity with a network is related to GM ODI. Therefore, it is possible that clusters demonstrating differential connectivity (in relation to ODI) with resting-state networks fall outside the main foci of a given network (Voets et al., 2012). Therefore, ODI may predict both intranetwork connectivity and internetwork connectivity. Although the implication of these relationships is still unclear, there is a growing interest in evaluating such between-network connections (Smith et al., 2013).

Our results suggest that resting-state networks are differentially affected by GM microstructure, in which task-negative and lower sensory/sensorimotor network connectivity associated positively with ODI, lateralized task-positive networks involved in higher cognition and attention remained unaffected or even decreased with higher ODI. These results align closely with network-specific effects of aging reported previously (Mowinckel et al., 2012). In previous endeavors linking brain structure to functional connectivity, long-range WM tracts have been a focus (Bullmore and Bassett, 2011). Assessment of GM microstructure, perhaps by indexing local circuitry and ensemble cytoarchitecture (Assaf et al., 2013), provides additional insight into structure-function relationships in the human brain (Bullmore and Bassett, 2011).

In terms of our diffusion image analysis approach, we used a conservative inclusion criterion (estimated GM fraction $>0.7$ ) 
Table 4. MNI coordinates and volume of clusters ( $p_{\text {FWE }}<0.05$ ) demonstrating effect of ODI extracted from each resting-state network (RSN) on its voxelwise functional connectivity (temporal coherence) while controlling for effects of age and sex (dual regression)

\begin{tabular}{|c|c|c|c|c|c|c|c|}
\hline \multirow[b]{2}{*}{ Cluster } & \multirow[b]{2}{*}{ Volume $\left(\mathrm{mm}^{3}\right)$} & \multirow[b]{2}{*}{ Minimum $p$-value } & \multicolumn{3}{|c|}{ MNI coordinates } & \multirow[b]{2}{*}{ Hemisphere } & \multirow[b]{2}{*}{ Brain regions } \\
\hline & & & $x$ & Y & Z & & \\
\hline \multicolumn{8}{|c|}{ Default mode network (ODI/RSN overlap ${ }^{a}: 9.0 \%, 0 \mathrm{DI} /$ age similarity $\left.{ }^{b}: 0.16\right)$} \\
\hline D1 & 174720 & 0.002 & 18 & -70 & 28 & Bilateral & $\begin{array}{l}\text { Cuneus, Lingual gyrus, } 0 c \mathrm{cPo}, \mathrm{LOC}, \text { Occipital Fus. } \\
\text { gyrus, Precuneus }\end{array}$ \\
\hline D2 & 67072 & 0.026 & -2 & 14 & 4 & Left & Caudate nucleus \\
\hline D3 & 3392 & 0.039 & 38 & 42 & -20 & Right & FPo \\
\hline \multicolumn{8}{|c|}{ Visual association network (ODI/RSN overlap: 8.5\%, 0DI/age similarity: 0.30 ) } \\
\hline V1 & 234944 & 0.003 & -10 & 38 & -12 & Bilateral & MedFC, ACC, FPo, ParaCG, OFC, IFG \\
\hline V2 & 15680 & 0.021 & 14 & -50 & -12 & Right & Lingual Gyrus, Fus. cortex \\
\hline V3 & 6272 & 0.021 & -50 & -6 & 44 & Left & $\operatorname{PreCG}$ \\
\hline V4 & 3904 & 0.021 & 14 & -62 & 32 & Right & Precuneus \\
\hline V5 & 3072 & 0.043 & 6 & -90 & 28 & Right & Cuneus, $0 c \mathrm{cPo}$ \\
\hline V6 & 2304 & 0.027 & 46 & 30 & 12 & Right & IFG \\
\hline V7 & 1600 & 0.029 & -22 & 26 & 28 & Left & MFG \\
\hline \multicolumn{8}{|c|}{ Sensorimotor network (ODI/RSN overlap: 30.0\%, 0DI/age similarity: 0.27 ) } \\
\hline S1 & 65728 & 0.02 & 30 & -22 & 20 & Right & Insula, Lingual gyrus, Fus. cortex \\
\hline S2 & 28544 & 0.026 & 2 & -18 & 44 & Bilateral & PCC, JLC, Precuneus \\
\hline S3 & 2176 & 0.04 & 2 & 38 & -4 & Bilateral & $\mathrm{ACC}$ \\
\hline S4 & 1984 & 0.034 & -10 & 46 & 28 & Left & Lingual gyrus, Fus. cortex \\
\hline S5 & 1856 & 0.037 & 62 & 14 & -8 & Right & TPo \\
\hline \multicolumn{8}{|c|}{ Primary visual network (ODI/RSN overlap: <1\%, 0DI/age similarity: 0.11 ) } \\
\hline P1 & 21504 & 0.021 & 22 & 18 & 8 & Right & Caudate Nucleus, Insula \\
\hline P2 & 14528 & 0.021 & 14 & 10 & 28 & Bilateral & $\mathrm{ACC}$ \\
\hline P3 & 9344 & 0.021 & -18 & -42 & 40 & Left & $\mathrm{PCC}$, Precuneus \\
\hline P4 & 3520 & 0.031 & -54 & 2 & 8 & Left & PreCG, Insula \\
\hline P5 & 2880 & 0.04 & -26 & 26 & 8 & Left & Insula \\
\hline \multicolumn{8}{|c|}{ Right frontotemporal network' (ODI/RSN overlap: $26.5 \%, 0 \mathrm{DI} /$ age similarity: no significant age effect) } \\
\hline R1 & 1600 & 0.03 & 22 & 50 & 24 & Right & FPo \\
\hline R2 & 768 & 0.027 & 50 & 34 & -8 & Right & FPo \\
\hline
\end{tabular}

ACC, anterior cingulate cortex; FOC, orbitofrontal cortex; FPo, frontal pole; Fus, fusiform; IFG, inferior frontal gyrus; JLC, juxtapositional cortex; LOC, lateral occipital cortex; MedFC, medial frontal cortex; MFG, middle frontal gyrus; OccPo, Occipital Pole; ParaCG, paracingulate gyrus; PreCG, precentral gyrus; PCC, posterior cingulate cortex.

${ }^{a}$ ODI/RSN overlap was calculated as the percentage of voxels demonstrating effect of orientation dispersion index (using a more liberal threshold: $p_{\mathrm{FWE}}<0.10$ ) that were located inside the main loci of the RSN ( $z>3$ ).

${ }^{b} \mathrm{ODI} /$ age spatial similarity was indexed using a Dice similarity score between the voxels demonstrating effect of orientation dispersion index and the voxels demonstrating effect of age (both thresholded at a more liberal $p_{\mathrm{FWE}}<0.10$ ). 'Except for right frontotemporal network, higher ODI was associated with increased functional connectivity.

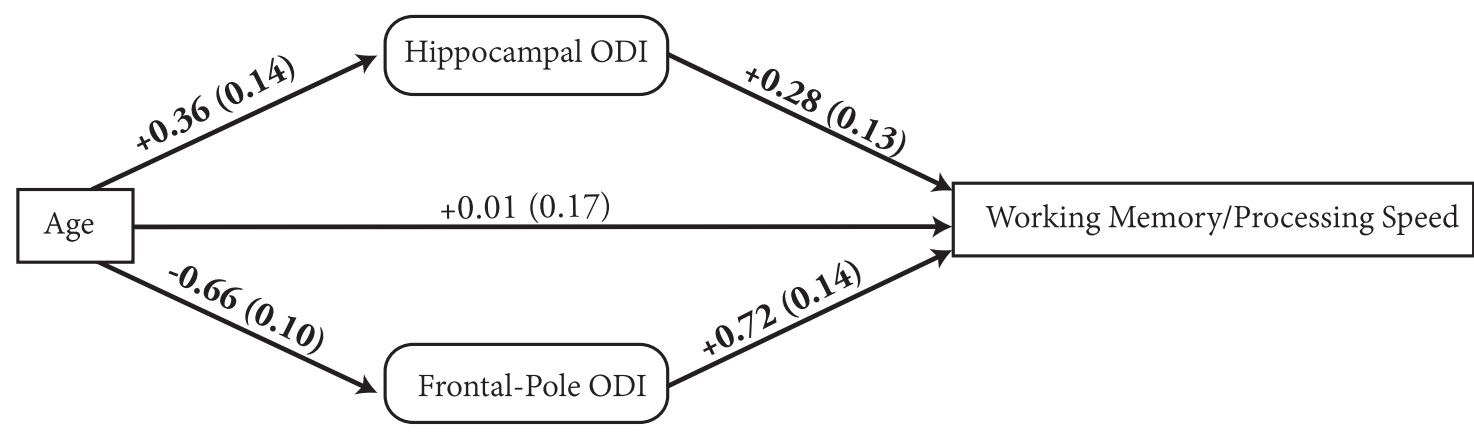

Figure 5. Mediation analysis of relationships among age (exogenous variable), frontal pole ODI, hippocampal ODI, and cognitive performance. Significant regression coefficients (SE) of associated paths are shown in boldface.

for voxels entering statistical analyses to further limit potential partial volume effects. However, this approach may inadvertently decrease statistical power in regions with thin cortical GM. Conversely, given our results, it appears that we had more than adequate power to achieve our stated aims. Even greater power is achievable with ultra-high-resolution diffusion-weighted MRI (Sotiropoulos et al., 2013). Our structure-function relationships were elicited using fMRI only in the resting condition. Future studies will have to be performed to broaden these findings and the potential effects of GM microstructure on the brain's functional dynamics during behavioral tasks and/or causal modeling of functional data (Friston et al., 2003) may provide additional useful knowledge. In addition, relaxation times $\left(\mathrm{T}_{1}\right.$ and $\left.\mathrm{T}_{2}\right)$ vary across brain tissues. This results in greater relative signal from the free water component (CSF) compared with other brain tissues given the same echo time. It has been shown that this issue may result in free water fraction overestimation in a bitensor model using single-shell diffusion data (Pasternak et al., 2009). Although $\mathrm{T}_{1}$-related effects tend to be minimal given the long TRs commonly used for diffusion imaging (as in our study: TR = $12,000 \mathrm{~ms}), \mathrm{T}_{2}$-related effects are more pronounced when TE is longer (Pasternak et al., 2009). However, to the best of our knowledge, no study to date has shown whether such biases exist for the NODDI model as well, which uses multishell diffusion data. In our study, CSF fraction estimates were only used to calculate the GM fraction and were not used as an outcome measure (Paster- 
nak et al., 2012). Therefore, we believe that any potential overestimation of the CSF-fraction would have minimal effect on the findings in our study.

Our cross-sectional design limits our ability to examine the temporal dynamics among aging, GM microstructural changes, and cognitive performance. Cohort effects and sampling biases can potentially confound effects of aging in studies using a crosssectional design. Previously published work has shown that a cross-sectional design can either overestimate (Nyberg et al., 2010) or underestimate (Raz et al., 2005) age-related changes in relational to longitudinal data. In this regard, our findings should be interpreted with caution because they are the first of their kind and our sample size can be considered relatively small. Although strong effects of age on GM ODI were detected, additional studies with larger sample sizes may detect relationships of smaller effect sizes and may allow for more complex multivariate modeling with age. Despite the agreement of our results with the postmortem literature, our interpretation of subcortical ODI changes as "compensatory" should be taken with caution. Tissue organization of subcortical regions that showed higher ODI with aging (i.e., three-layered cerebellar cortex and hippocampus) are considerably different from that of neocortex, which has a six-layered architecture (Shepherd, 2011; Roostaei et al., 2014). Therefore, an alternative interpretation is that age-related ODI differences in these structures represent different underlying microstructural phenomena.

Our findings provide evidence for vulnerability and compensatory neural mechanisms of cognitive aging in GM microstructure in vivo that have functional and cognitive impact. Microstructural GM changes represent a new target of investigation, not only for aging, but also for brain disorders with known dendritic susceptibility such as Alzheimer's disease (Penzes et al., 2011). These alterations may serve as either a diagnostic marker for at the earliest stage of illness or as targets for therapeutic intervention.

\section{References}

Anderson B, Rutledge V (1996) Age and hemisphere effects on dendritic structure. Brain 119:1983-1990. CrossRef Medline

Assaf Y, Alexander DC, Jones DK, Bizzi A, Behrens TE, Clark CA, Cohen Y, Dyrby TB, Huppi PS, Knoesche TR, Lebihan D, Parker GJ, Poupon C, Poupon C, Anaby D, Anwander A, Bar L, Barazany D, Blumenfeld-Katzir T, De-Santis S, Duclap D, Figini M, Fischi E, Guevara P, Hubbard P, Hofstetter S, Jbabdi S, Kunz N, Lazeyras F, Lebois A, Liptrot MG, Lundell H, Mangin JF, Dominguez DM, Morozov D, Schreiber J, Seunarine K, Nava S, Poupon C, Riffert T, Sasson E, Schmitt B, Shemesh N, Sotiropoulos SN, Tavor I, Zhang HG, Zhou FL (2013) The CONNECT project: combining macro-and micro-structure. Neuroimage 80:273-282. CrossRef Medline

Avants BB, Tustison NJ, Wu J, Cook PA, Gee JC (2011a) An open source multivariate framework for $\mathrm{n}$-tissue segmentation with evaluation on public data. Neuroinformatics 9:381-400. CrossRef Medline

Avants BB, Tustison NJ, Song G, Cook PA, Klein A, Gee JC (2011b) A reproducible evaluation of ANTs similarity metric performance in brain image registration. Neuroimage 54:2033-2044. CrossRef Medline

Ball G, Srinivasan L, Aljabar P, Counsell SJ, Durighel G, Hajnal JV, Rutherford MA, Edwards AD (2013) Development of cortical microstructure in the preterm human brain. Proc Natl Acad Sci U S A 110:9541-9546. CrossRef Medline

Beckmann CF, Smith SM (2004) Probabilistic independent component analysis for functional magnetic resonance imaging. IEEE Trans Med Imaging 23:137-152. CrossRef Medline

Beckmann CF, DeLuca M, Devlin JT, Smith SM (2005) Investigations into resting-state connectivity using independent component analysis. Philos Trans R Soc Lond B Biol Sci 360:1001-1013. CrossRef Medline

Buell SJ, Coleman PD (1979) Dendritic growth in the aged human brain and failure of growth in senile dementia. Science 206:854-856. CrossRef Medline

Bullmore ET, Bassett DS (2011) Brain graphs: graphical models of the human brain connectome. Annu Rev Clin Psychol 7:113-140. CrossRef Medline

Chen S, Hillman DE (1999) Dying-back of Purkinje cell dendrites with synapse loss in aging rats. J Neurocytol 28:187-196. CrossRef Medline

Dale AM, Fischl B, Sereno MI (1999) Cortical surface-based analysis: I. Segmentation and surface reconstruction. Neuroimage 9:179-194. CrossRef Medline

de Brabander JM, Kramers RJ, Uylings HB (1998) Layer-specific dendritic regression of pyramidal cells with ageing in the human prefrontal cortex. Eur J Neurosci 10:1261-1269. CrossRef Medline

Dickstein DL, Weaver CM, Luebke JI, Hof PR (2013) Dendritic spine changes associated with normal aging. Neuroscience 251:21-32. CrossRef Medline

Duan H, Wearne SL, Rocher AB, Macedo A, Morrison JH, Hof PR (2003) Age-related dendritic and spine changes in corticocortically projecting neurons in macaque monkeys. Cereb Cortex 13:950-961. CrossRef Medline

Ferreira LK, Busatto GF (2013) Resting-state functional connectivity in normal brain aging. Neurosci Biobehav Rev 37:384-400. CrossRef Medline

Filippini N, MacIntosh BJ, Hough MG, Goodwin GM, Frisoni GB, Smith SM, Matthews PM, Beckmann CF, Mackay CE (2009) Distinct patterns of brain activity in young carriers of the APOE- $\varepsilon 4$ allele. Proc Natl Acad Sci U S A 106:7209-7214. CrossRef Medline

Fischl B (2012) FreeSurfer. Neuroimage 62:774-781. CrossRef Medline

Fischl B, Dale AM (2000) Measuring the thickness of the human cerebral cortex from magnetic resonance images. Proc Natl Acad Sci U S A 97: 11050-11055. CrossRef Medline

Flood DG, Buell SJ, Defiore CH, Horwitz GJ, Coleman PD (1985) Agerelated dendritic growth in dentate gyrus of human brain is followed by regression in the 'oldest old'. Brain Res 345:366-368. CrossRef Medline

Friston KJ, Harrison L, Penny W (2003) Dynamic causal modelling. Neuroimage 19:1273-1302. CrossRef Medline

Glasser MF, Van Essen DC (2011) Mapping human cortical areas in vivo based on myelin content as revealed by T1-and T2-weighted MRI. J Neurosci 31:11597-11616. CrossRef Medline

Griffanti L, Salimi-Khorshidi G, Beckmann CF, Auerbach EJ, Douaud G, Sexton CE, Zsoldos E, Ebmeier KP, Filippini N, Mackay CE, Moeller S, Xu J, Yacoub E, Baselli G, Ugurbil K, Miller KL, Smith SM (2014) ICAbased artefact removal and accelerated fMRI acquisition for improved resting state network imaging. Neuroimage 95:232-247. CrossRef Medline

Grydeland H, Walhovd KB, Tamnes CK, Westlye LT, Fjell AM (2013) Intracortical myelin links with performance variability across the human lifespan: results from T1-and T2-weighted MRI myelin mapping and diffusion tensor imaging. J Neurosci 33:18618-18630. CrossRef Medline

Jacobs B, Scheibel AB (1993) A quantitative dendritic analysis of Wernicke's area in humans. I. Lifespan changes. J Comp Neurol 327:83-96. CrossRef Medline

Jacobs B, Driscoll L, Schall M (1997) Life-span dendritic and spine changes in areas 10 and 18 of human cortex: A quantitative Golgi study. J Comp Neurol 386:661-680. CrossRef Medline

Jenkinson M, Beckmann CF, Behrens TE, Woolrich MW, Smith SM (2012) Fsl. Neuroimage 62:782-790. CrossRef Medline

Jespersen SN, Kroenke CD, Østergaard L, Ackerman JJ, Yablonskiy DA (2007) Modeling dendrite density from magnetic resonance diffusion measurements. Neuroimage 34:1473-1486. CrossRef Medline

Kim SY, Chung K, Deisseroth K (2013) Light microscopy mapping of connections in the intact brain. Trends Cogn Sci 17:596-599. CrossRef Medline

Koo BB, Hua N, Choi CH, Ronen I, Lee JM, Kim DS (2009) A framework to analyze partial volume effect on gray matter mean diffusivity measurements. Neuroimage 44:136-144. CrossRef Medline

Kumazawa S, Yoshiura T, Honda H, Toyofuku F, Higashida Y (2010) Partial volume estimation and segmentation of brain tissue based on diffusion tensor MRI. Med Phys 37:1482-1490. CrossRef Medline

Liu T, Li H, Wong K, Tarokh A, Guo L, Wong ST (2007) Brain tissue segmentation based on DTI data. Neuroimage 38:114-123. CrossRef Medline 
Miller MD, Paradis CF, Houck PR, Mazumdar S, Stack JA, Rifai AH, Mulsant B, Reynolds CF 3rd (1992) Rating chronic medical illness burden in geropsychiatric practice and research: application of the Cumulative Illness Rating Scale. Psychiatry Res 41:237-248. <zbap $><$ : pdfs\%3B12\%3Bhttp://dx.doi.org/10.1016/0165-1781\%3B/Border

$>$ http://dx.doi.org/10.1016/0165-1781<: pdfe\%3B12><zbapx $>(92) 90005-\mathrm{N}$ Medline

Mowinckel AM, Espeseth T, Westlye LT (2012) Network-specific effects of age and in-scanner subject motion: a resting-state fMRI study of 238 healthy adults. Neuroimage 63:1364-1373. CrossRef Medline

Nakamura S, Akiguchi I, Kameyama M, Mizuno N (1985) Age-related changes of pyramidal cell basal dendrites in layers III and V of human motor cortex: a quantitative Golgi study. Acta Neuropathol 65:281-284. CrossRef Medline

Nichols TE, Holmes AP (2002) Nonparametric permutation tests for functional neuroimaging: a primer with examples. Hum Brain Mapp 15:1-25. CrossRef Medline

Nyberg L, Salami A, Andersson M, Eriksson J, Kalpouzos G, Kauppi K, Lind J, Pudas S, Persson J, Nilsson LG (2010) Longitudinal evidence for diminished frontal cortex function in aging. Proc Natl Acad Sci U S A 107: 22682-22686. CrossRef Medline

Oldfield RC (1971) The assessment and analysis of handedness: the Edinburgh inventory. Neuropsychologia 9:97-113. CrossRef Medline

Pasternak O, Sochen N, Gur Y, Intrator N, Assaf Y (2009) Free water elimination and mapping from diffusion MRI. Magn Reson Med 62:717-730. CrossRef Medline

Pasternak O, Westin CF, Bouix S, Seidman LJ, Goldstein JM, Woo TU, Petryshen TL, Mesholam-Gately RI, McCarley RW, Kikinis R, Shenton ME, Kubicki M (2012) Excessive extracellular volume reveals a neurodegenerative pattern in schizophrenia onset. J Neurosci 32:17365-17372. CrossRef Medline

Penzes P, Cahill ME, Jones KA, VanLeeuwen JE, Woolfrey KM (2011) Dendritic spine pathology in neuropsychiatric disorders. Nat Neurosci 14: 285-293. CrossRef Medline

Pereira JB, Valls-Pedret C, Ros E, Palacios E, Falcón C, Bargalló N, BartrésFaz D, Wahlund LO, Westman E, Junque C (2014) Regional vulnerability of hippocampal subfields to aging measured by structural and diffusion MRI. Hippocampus 24:403-414. CrossRef Medline

Preacher KJ, Hayes AF (2008) Asymptotic and resampling strategies for assessing and comparing indirect effects in multiple mediator models. Behav Res Methods 40:879-891. CrossRef Medline

Pyapali GK, Turner DA (1996) Increased dendritic extent in hippocampal CA1 neurons from aged F344 rats. Neurobiol Aging 17:601-611. CrossRef Medline

Rathi Y, Pasternak O, Savadjiev P, Michailovich O, Bouix S, Kubicki M, Westin CF, Makris N, Shenton ME (2014) Gray matter alterations in early aging: A diffusion magnetic resonance imaging study. Hum Brain Mapp 35:3841-3856. CrossRef Medline

Raz N, Lindenberger U, Rodrigue KM, Kennedy KM, Head D, Williamson A, Dahle C, Gerstorf D, Acker JD (2005) Regional brain changes in aging healthy adults: general trends, individual differences and modifiers. Cereb Cortex 15:1676-1689. CrossRef Medline

Reisberg B, Franssen EH, Hasan SM, Monteiro I, Boksay I, Souren LEM, Kenowsky S, Auer SR, Elahi S, Kluger A (1999) Retrogenesis: clinical, physiologic, and pathologic mechanisms in brain aging, Alzheimer's and other dementing processes. Eur Arch Psychiatry Clin Neurosci 249:S28S36. CrossRef Medline

Reitan RM, Wolfson D (1985) The Halstead-Reitan neuropsychological test battery: theory and clinical interpretation: Neuropsychology Tucson, AZ.

Rogers J, Zornetzer SF, Bloom FE, Mervis RE (1984) Senescent microstructural changes in rat cerebellum. Brain Res 292:23-32. CrossRef Medline

Roostaei T, Nazeri A, Sahraian MA, Minagar A (2014) The human cerebel- lum: a review of physiologic neuroanatomy. Neurol Clin 32:859-869. CrossRef Medline

Salimi-Khorshidi G, Douaud G, Beckmann CF, Glasser MF, Griffanti L, Smith SM (2014) Automatic denoising of functional MRI data: combining independent component analysis and hierarchical fusion of classifiers. Neuroimage 90:449-468. CrossRef Medline

Schmahmann JD, Pandya DN (1997) The cerebrocerebellar system. Int Rev Neurobiol 41:31-60. CrossRef Medline

Schwarz CG, Reid RI, Gunter JL, Senjem ML, Przybelski SA, Zuk SM, Whitwell JL, Vemuri P, Josephs KA, Kantarci K, Thompson PM, Petersen RC, Jack CR Jr; Alzheimer's Disease Neuroimaging Initiative (2014) Improved DTI registration allows voxel-based analysis that outperforms Tract-Based Spatial Statistics. Neuroimage 94:65-78. CrossRef Medline

Shepherd GM (2011) The microcircuit concept applied to cortical evolution: from three-layer to six-layer cortex. Front Neuroanat 5.

Smith SM (2002) Fast robust automated brain extraction. Hum Brain Mapp 17:143-155. CrossRef Medline

Smith SM, Nichols TE (2009) Threshold-free cluster enhancement: addressing problems of smoothing, threshold dependence and localisation in cluster inference. Neuroimage 44:83-98. CrossRef Medline

Smith SM, Jenkinson M, Johansen-Berg H, Rueckert D, Nichols TE, Mackay CE, Watkins KE, Ciccarelli O, Cader MZ, Matthews PM, Behrens TE (2006) Tract-based spatial statistics: voxelwise analysis of multi-subject diffusion data. Neuroimage 31:1487-1505. CrossRef Medline

Smith SM, Fox PT, Miller KL, Glahn DC, Fox PM, Mackay CE, Filippini N, Watkins KE, Toro R, Laird AR, Beckmann CF (2009) Correspondence of the brain's functional architecture during activation and rest. Proc Natl Acad Sci U S A 106:13040-13045. CrossRef Medline

Smith SM, Vidaurre D, Beckmann CF, Glasser MF, Jenkinson M, Miller KL, Nichols TE, Robinson EC, Salimi-Khorshidi G, Woolrich MW, Barch DM, Uğurbil K, Van Essen DC (2013) Functional connectomics from resting-state fMRI. Trends Cogn Sci 17:666-682. CrossRef Medline

Sotiropoulos SN, Behrens TE, Jbabdi S (2012) Ball and rackets: inferring fiber fanning from diffusion-weighted MRI. Neuroimage 60:1412-1425. CrossRef Medline

Sotiropoulos SN, Jbabdi S, Xu J, Andersson JL, Moeller S, Auerbach EJ, Glasser MF, Hernandez M, Sapiro G, Jenkinson M, Yacoub E, Lenglet C, Van Essen DC, Ugurbil K, Behrens TE, Behrens TE (2013) Advances in diffusion MRI acquisition and processing in the Human Connectome Project. Neuroimage 80:125-143. CrossRef Medline

Spruston N (2008) Pyramidal neurons: dendritic structure and synaptic integration. Nat Rev Neurosci 9:206-221. CrossRef Medline

Trenerry MR, Crosson B, DeBoe J, Leber WR (1989) Stroop neuropsychological screening test manual. Odessa, FL: Psychological Assessment Resources.

Viswanathan A, Freeman RD (2007) Neurometabolic coupling in cerebral cortex reflects synaptic more than spiking activity. Nat Neurosci 10:1308 1312. CrossRef Medline

Voets NL, Beckmann CF, Cole DM, Hong S, Bernasconi A, Bernasconi N (2012) Structural substrates for resting network disruption in temporal lobe epilepsy. Brain 135:2350-2357. CrossRef Medline

Wechsler D (1997) The Wechsler Memory Scale. San Antonio, TX: Psychological Corporation, Harcourt Brace Jovanovich.

Winston GP, Micallef C, Symms MR, Alexander DC, Duncan JS, Zhang H (2014) Advanced diffusion imaging sequences could aid assessing patients with focal cortical dysplasia and epilepsy. Epilepsy Res 108:336339. CrossRef Medline

Young ME, Ohm DT, Dumitriu D, Rapp PR, Morrison JH (2014) Differential effects of aging on dendritic spines in visual cortex and prefrontal cortex of the rhesus monkey. Neuroscience 274:33-43. CrossRef Medline

Zhang H, Schneider T, Wheeler-Kingshott CA, Alexander DC (2012) NODDI: practical in vivo neurite orientation dispersion and density imaging of the human brain. Neuroimage 61:1000-1016. CrossRef Medline 